\title{
Prevention of mother-to-child transmission of HIV-I through breastfeeding by treating infants or mothers prophylactically with antiretrovirals in Dar es Salaam, Tanzania: the MITRA and MITRA PLUS studies
}

\author{
Charles Kilewo*1, Katarina Karlsson², Matilda Ngarina3, \\ Augustine Massawe ${ }^{4}$, Eligius Lyamuya ${ }^{5}$, Rosina Lipyoga ${ }^{3}$, Georgina Msemo ${ }^{6}$, \\ Muhamad Bakari ${ }^{7}$, Andrew Swai ${ }^{8}$, Fred Mhalu ${ }^{5}$ and Gunnel Biberfeld ${ }^{2}$
}

\begin{abstract}
Address: ${ }^{1}$ Department of Obstetrics and Gynaecology, Muhimbili University of Health and Allied Sciences, Dar es Salaam, Tanzania, ${ }^{2}$ Department of Immunology and Vaccinology, Swedish Institute for Infectious Disease Control (SMI) and Department of Microbiology, Tumor and Cell Biology, Karolinska Institute, SE-171 82, Stockholm, Sweden, ${ }^{3}$ Department of Obstetrics and Gynaecology, Muhimbili National Hospital, Dar es Salaam, Tanzania, ${ }^{4}$ Department of Pediatrics, Muhimbili University of Health and Allied Sciences, Dar es Salaam, Tanzania, ${ }^{5}$ Department of Microbiology and Immunology, Muhimbili University of Health and Allied Sciences, Dar es Salaam, Tanzania, ${ }^{6}$ Department of Pediatrics, Muhimbili National Hospital, Dar es Salaam, Tanzania, ${ }^{7}$ Department of Internal Medicine, Muhimbili University of Health and Allied Sciences, Dar es Salaam, Tanzania and ${ }^{8}$ Department of Internal Medicine, Muhimbili National Hospital, Dar es Salaam, Tanzania

* Corresponding author
\end{abstract}

from Fourth Dominique Dormont International Conference. Host-Pathogen Interactions in Chronic Infections Paris, France. 13-15 December 2007

Published: 9 April 2008

Retrovirology 2008, 5(Suppl I):OI7 doi:I0.II86/I742-4690-5-SI-OI7

This abstract is available from: http://www.retrovirology.com/content/5/SI/OI7

C 2008 Kilewo et al.; licensee BioMed Central Ltd.

\section{Background}

Short course antiretroviral (ARV) treatment around delivery reduces early mother-to-child transmission (MTCT) of HIV-1 by 35-60 \%. However, additional interventions are required to prevent postnatal transmission through breastfeeding. We have performed two studies (MITRA and MITRA PLUS) to investigate the possibility to reduce MTCT of HIV-1 by prophylactic ARV treatment of infants or mothers during breastfeeding.

\section{Materials and methods}

In the MITRA study HIV-1 infected pregnant women were treated during late pregnancy and for one week after delivery with two ARV drugs, zidovudine (ZDV) and lamivudine (3TC). Infants were treated with these drugs for one week after birth and then with 3TC alone during breastfeeding (maximum 6 months). In the MITRA PLUS study the HIV-1 infected mothers were treated with three ARV drugs, $\mathrm{ZDV}+3 \mathrm{TC}+$ nevirapine (NVP) during late pregnancy and breastfeeding (NVP was replaced by nelfinavir for mothers with adverse reactions on NVP). Treatment of the mothers was stopped at six months except for those who needed ARV treatment for their own health. In both studies mothers were counseled on exclusive breastfeeding and encouraged to stop at six months. Transmission of HIV-1 was analyzed using the Kaplan Meier survival technique.

\section{Results}

In the MITRA study 398 infants were included in the transmission analysis. The cumulative proportion of HIVinfected infants was 3.8\% (95\%CI 2.0\%-5.6\%) at 6 weeks and $4.9 \%$ (95\%CI $2.7 \%-7.1 \%)$ at 6 months of age. In the MITRA PLUS study there were 440 infants included in the transmission analysis. The proportion of HIV-1 infected infants was $4.1 \%(95 \%$ CI $2.1 \%-6.0 \%)$ at 6 weeks and $5.0 \%$ (95\%CI 3.2\%-7.0\%) at 6 months. The median time of breastfeeding was 18 weeks in the MITRA study and 24 weeks in the MITRA PLUS study. NVP-related skin reactions occurred in 24 (5.5\%) of 433 NVP-treated women, of whom 6 had Steven Johnson syndrome. All women with skin reactions had CD4 cell counts $>200 / \mathrm{mm}^{3}$. 


\section{Conclusions}

The HIV-1 transmission rates at 6 weeks and 6 months after delivery in the MITRA and MITRA PLUS studies were similar and are among the lowest reported in a breastfeeding population in sub-Saharan Africa. The strategy used in the MITRA PLUS study is the obvious choice for mothers who need ARV treatment for their own health whereas prophylactic ARV treatment of the infant during breastfeeding could be a possible choice for mothers with high CD4 cell counts.

Publish with Biomed Central and every scientist can read your work free of charge

"BioMed Central will be the most significant development for disseminating the results of biomedical research in our lifetime. " Sir Paul Nurse, Cancer Research UK

Your research papers will be:

- available free of charge to the entire biomedical community

- peer reviewed and published immediately upon acceptance

- cited in PubMed and archived on PubMed Central

- yours - you keep the copyright 